\title{
Tanshinone IIA Could Inhibit Pancreatic Cancer BxPC-3 Cells through Increasing PERK, ATF6, Caspase-12 and CHOP Expression to Induce Apoptosis
}

\author{
Chin-Cheng Su Su,2,3 $^{1,3}$ \\ ${ }^{1}$ Tumor Research Center of Integrative Medicine, Changhua Christian Hospital, Changhua, China \\ ${ }^{2}$ Department of Surgery, Changhua Christian Hospital, Changhua, China \\ ${ }^{3}$ Comprehensive Breast Cancer Center, Changhua Christian Hospital, Changhua, China \\ ${ }^{4}$ School of Chinese Medicine, College of Chinese Medicine, China Medical University, Taichung, China \\ Email: succ.maeva@msa.hinet.net
}

Received 8 February 2015; accepted 12 March 2015; published 17 March 2015

Copyright (C) 2015 by author and Scientific Research Publishing Inc.

This work is licensed under the Creative Commons Attribution International License (CC BY).

http://creativecommons.org/licenses/by/4.0/

(c) (i) Open Access

\begin{abstract}
Tanshinone IIA (Tan-IIA) is extracted from Dan-Shen. Tan-IIA could inhibit human pancreatic cancer BxPC-3 cells through decreasing TCTP, Mcl-1 and Bcl-xl expression in vitro. Our previous study showed that Tan-IIA can inhibit hepatocellular carcinoma hep-J5 cells and human breast cancer BT-20 cells through inducing endoplasmic reticulum (ER) stress. In the present study, we investigated the ER stress related protein expressions in human pancreatic cancer BXPC3 cells were treated with Tan-IIA. The ER stress related protein expressions in human pancreatic cancer BxPC-3 cells were evaluated by western blotting. The results showed that Tan-IIA can increase the protein expressions of PERK, ATF6, Caspase-12 and CHOP, but decrease Bip, PDI, Calnexin, Calreticulin and Bcl-2 expression. These findings indicated that Tan-IIA can inhibit human pancreatic cancer BxPC-3 cells by inducing ER stress to induce apoptosis.
\end{abstract}

\section{Keywords}

Tanshinone IIA, Pancreatic Cancer BxPC-3 Cells, ER Stress, Apoptosis

\section{Introduction}

In 2012, pancreatic cancer is the 4th leading cause of cancer death in the US [1]. Gemcitabine is the standard treatment for pancreatic cancer patients, but the survival rate for one year was only $18 \%$ [2]. Although many ef-

How to cite this paper: Su, C.-C. (2015) Tanshinone IIA Could Inhibit Pancreatic Cancer BxPC-3 Cells through Increasing PERK, ATF6, Caspase-12 and CHOP Expression to Induce Apoptosis. J. Biomedical Science and Engineering, 8, 149-159. 
forts have been made to improve the clinical efficacy, but current chemotherapeutic medicines for pancreatic cancer are unsatisfactory [3]-[5] and need to identify new treatments. Tanshinone IIA $\left(\mathrm{C}_{19} \mathrm{H}_{18} \mathrm{O}_{3}\right)$ is one of the active components in Radix Salviae miltiorrhizae [6] [7]. It was well documented that Tanshinone IIA (Tan-IIA) has anti-inflammatory activities [8] [9] and antioxidant properties [10] [11]. It was well documented that TanIIA can inhibit many human cancer cell lines through different molecular mechanisms, such as Tan-IIA downregulates ErbB-2 and up-regulates TNF-alpha expression to inhibit colon cancer colo205 cells [12], Tanshinone IIA inhibits human breast cancer MDA-MB-231 cells through increasing Bax to Bcl-xL ratios [13], Tanshinone IIA inhibits human non-small cell lung cancer A549 cells through the induction of reactive oxygen species and decreasing the mitochondrial membrane potential [14], Tan-IIA could inhibit small cell lung cancer H146 cells by up-regulating the Bax/Bcl-2 ratio and decreasing mitochondrial membrane potential [15], Tan-IIA possesses cytotoxic effects in human pancreatic (MIAPaCa-2) tumor cell lines was documented, but the mechanism has not been established [16]. Because our previous studies showed Tan-IIA could inhibit hepatocellular carcinoma Hep-J5 cells [17] and breast cancer BT-20 cells [18] through inducing ER stress. Our previous study also showed Tan-IIA can inhibit human pancreatic cancer BxPC-3 cells through decreasing TCTP, Mcl-1 and Bcl-xl expression in vitro [19]. In the present study, we investigated the ER stress related protein expressions in human pancreatic cancer BxPC3 cells were treated with Tan-IIA.

\section{Materials and Methods}

Chemicals and reagents: Tan-IIA (molecular formula: C19H18O3) was purchased from Sigma (CAS-No 56872-9). The BxPC-3 human pancreatic cancer cell line (BCRC NUMBER: 60283) was obtained from the Food Industry Research and Development Institute (Hsinchu, Taiwan). MTT [3-(4,5-dimethylthiazol-2-y1)-2,5-diphenyltetrazolium bromide], Sodium deoxycholate, leupeptin, Triton X-100, Tris- $\mathrm{HCl}$, ribonuclease-A and sodium orthovanadate were obtained from Sigma Chemical Co. (St. Louis, MO, USA). Dimethyl sulfoxide (DMSO), potassium phosphates and TE buffer were purchased from Merck Co. (Darmstadt, Germany). Fetal bovine serum (FBS) and glutamine were obtained from Gibco BRL (Grand Island, NY, USA). Sodium pyruvate, HEPES, RPMI-1640, trypsin-EDTA, mouse anti- $\beta$-actin, and penicillin-streptomycin, were obtained from Sigma-Aldrich (St. Louis, MO, USA). Buffer (10X TG-SDS), Tween-20 and glycine, were obtained from Amresco (St. Louis, MO, USA). BioMax film was obtained from Kodak. Antibodies BcL-2 (cell signaling \#2872, MW 28 kDa), Bip (cell signaling \#9956, MW $78 \mathrm{kDa}$ ), PDI (cell signaling \#9956, MW $57 \mathrm{kDa}$ ), Calnexin (cell signaling \#9956, MW $90 \mathrm{kDa}$ ), Caspase 9 (cell signaling \#9508, MW $37 \mathrm{kDa}$ ), C/EBP-homologous protein (CHOP; also known as GADD153) (cell signaling \#9956, MW $27 \mathrm{kDa}$ ): protein kinase RNA (PKR)-like ER kinase (PERK) (cell signaling \#9956, MW 140 kDa), linositol-requiring protein kinase RNA (PKR)-like ER kinase (ein-1 IRE1 $\alpha$ ) (cell signaling \#9956, MW $130 \mathrm{kDa}$ ), p-JNK (cell signaling \#9910, MW $46 \mathrm{kDa}$ ) were obtained from cell signaling. Caspase 12 (abcam, ab62484, MW 38 kDa), elF2 $\alpha$ (abcam, ab5369, MW 36 kDa), ATF4 (abcam, ab1370, MW 38 kDa): activating transcription factor-6 (ATF6) (abcam, ab37149, MW 50 kDa) were obtained from abcam. Calreticulin (GeneTex, GTX42683, MW $48 \mathrm{kDa}$ ) was obtained from GeneTex. Other materials and reagents not specified were obtained from Sigma or Merck.

Cell culture: The BxPC-3 cells are established from a 61-year-old female pancreatic epithelial cell adenocarcinoma was obtained from the Food Industry Research and Development Institute (Hsinchu, Taiwan), were maintained in RPMI-1640 medium containing 10\% FBS, 1\% penicillin/streptomycin (10,000 U/ml penicillin, 10 $\mathrm{mg} / \mathrm{ml}$ streptomycin) at $37^{\circ} \mathrm{C}$ in a humidified atmosphere containing $5 \% \mathrm{CO}_{2}$.

\subsection{Cytotoxicity Assay}

The cytotoxicity of Tan-IIA for BxPC-3 cells was evaluated by MTT assay in triplicate as document described [20]. Briefly, the BxPC-3 cells were plated in 96-well plates at a density of $1 \times 10^{4}$ cells/well and treated with various concentrations of Tan-IIA for different durations (24, 48 and 72 hours). Subsequently, the cells were incubated with $100 \mu \mathrm{l}$ MTT $(1 \mathrm{mg} / \mathrm{ml})$ in fresh complete RPMI medium for $2 \mathrm{hrs}$. The surviving cells converted MTT to formazan by forming a blue-purple color when dissolved in dimethyl sulfoxide. Absorbance was measured using an ELISA microplate reader at $590 \mathrm{~nm}$. The relative percentage of cell viability was calculated by dividing the absorbance of treated cells by that of the control in each experiment, using the following formula: Proliferation rate $(\%)=($ ODtest - ODblank $) \times 100$, where ODtest and ODblank are the optical density of the test substances and the blank control, respectively. 


\subsection{Western Blot}

The effects of Tan-IIA on the protein expressions of Bip, PDI, Calnexin, Calreticulin, IRE1 $\alpha$, PERK, elF2 $\alpha$, ATF6, ATF4, Caspase-12, Caspase-9, CHOP and Bcl-2 in BxPC-3 cells

BxPC-3 cells were treated with various concentrations $(0,4.2$ and $8.5 \mu \mathrm{M})$ of Tan-IIA for 24 hours and the proteins expression levels of Bip, PDI, Calnexin, Calreticulin, IRE1 $\alpha$, PERK, elF2 $\alpha$, ATF6, ATF4, Caspase-12, Caspase-9, CHOP and Bcl-2 were evaluated by western blotting.

BxPC-3 cells were treated with various concentrations $(0,2.0$ and $4.0 \mu \mathrm{M})$ of Tan-IIA for 48 hours and the proteins expression levels of Bip, PDI, Calnexin, Calreticulin, IRE1 $\alpha$, PERK, elF2 $\alpha$, ATF6, ATF4, Caspase-12, Caspase-9, CHOP and Bcl-2 were evaluated by western blotting.

BxPC-3 cells were treated with Tan-IIA $(8.5 \mu \mathrm{M})$ for different durations $(0,24,48$ and $72 \mathrm{~h})$ and then the proteins expression levels of Bip, PDI, Calnexin, Calreticulin, IRE1 $\alpha$, PERK, elF2 $\alpha$, ATF6, ATF4, Caspase-12, Caspase-9, CHOP and Bcl-2 were evaluated by western blotting. BxPC-3 cells were treated with Tan-IIA (8.5 $\mu \mathrm{M})$ for different durations $(0,2,4,6,8,12,24$ and $48 \mathrm{~h})$ and then the proteins expression levels of Bip, PDI, Calnexin, Calreticulin were evaluated by western blotting. The western blot procedures as document described [21] [22]. Briefly, BxPC-3 cells were treated with various concentrations of Tan-IIA for different durations; the cells were lysed in the ice-cold whole cell extract buffer containing the protease inhibitors. The lysate was vibrated for $30 \mathrm{~min}$ at $4^{\circ} \mathrm{C}$ and centrifuged at 10,000 rpm for 10 minutes. Protein concentration was measured by BCA protein assay kit (Pierce, Rockford, IL). Equal amounts of proteins $(40 \mu \mathrm{g})$ were subjected to electrophoresis using $10 \%$ - 15\% sodium dodecyl sulfate-polyacrylamide gels. To verify equal protein loading and transfer, proteins were then transferred to polyvinylidene difluoride membranes and then the membranes were blocked overnight at $4^{\circ} \mathrm{C}$ using blocking buffer ( $5 \%$ non-fat dried milk in solution containing $50 \mathrm{mM}$ Tris/ $\mathrm{HCl}(\mathrm{pH} 8.0)$, $2 \mathrm{mM} \mathrm{CaCl}_{2}, 80 \mathrm{mM}$ sodium chloride, $0.05 \%$ Tween 20 and $0.02 \%$ sodium azide). The membranes were then incubated with specific primary antibody. After incubated with primary Abs for 2 hours at $25^{\circ} \mathrm{C}$, the membranes were washed thrice with TBST buffer and followed by anti-rabbit or anti-mouse immunoglobulin G-horseradish peroxidase conjugated secondary antibodies. The membranes were washed three times for $10 \mathrm{~min}$ with washing solution. Finally, the immunoreactive protein bands were visualized on the X-ray film and analyzed using the enhanced chemiluminescence detection system (Perkin Elmer Life and Analytical Sciences, Boston, MA). The detection of $\beta$-actin was used as an internal control in all of the data for Western blotting.

\subsection{Immunocytochemistry Staining}

The cells were cultured in 6-well plate dish at a density of $5 \times 10^{5}$ per well for 16 - 20 hours. After Tan-IIA treatment for 24 or 48 hours, the cells were washed with PBS. Then fixation with $50 \%$ acetone and $50 \%$ methanol solution overnight at $4^{\circ} \mathrm{C}$, the cells were washed three times with PBS, and non-specific binding sites were blocked in PBS containing $0.1 \%$ BSA for $1 \mathrm{~h}$ at room temperature. Thereafter, the cells were separately incubated with rabbit anti-caspase $3(1: 20)$ antibody in PBS containing $0.1 \%$ BSA overnight at $4{ }^{\circ} \mathrm{C}$, and washed three times with PBS. Then the cells were incubated with anti-rabbit FITC (1:200) in PBS containing 0.1\% BSA for $1 \mathrm{~h}$ at room temperature, and washed three times with PBS. The nuclei were stained with the $5 \mu \mathrm{g} / \mathrm{ml}$ PI, respectively. After staining, the samples were immediately examined under Olympus IX81 microscope (Olympus, Tokyo, Japan).

\subsection{Statistical Analysis}

Values are presented as mean \pm SD. The student's t-test was used to analyze the statistical significance. ${ }^{*} \mathrm{p}$ value $<$ 0.05 was considered significant for all tests.

\section{Results}

\subsection{The Cytotoxicity of Tan-IIA in BxPC-3 Cells}

BxPC-3 cells were cultured with various concentrations $(0,3,10,31$ and $51 \mu \mathrm{M})$ of Tan-IIA for different durations (24, 48 and 72 hours). The results showed that the viable cell percentages relative to the control were 78.81 $\pm 1.050,48.43 \pm 0.773,30.78 \pm 1.202$, $23.58 \pm 0.716$ for 24 hours; $55.90 \pm 1.422$, $31.32 \pm 1.215,15.83 \pm 0.389$, $16.04 \pm 3.971$ for 48 hours; $40.81 \pm 0.874,19.33 \pm 0.348,9.04 \pm 0.268,3.71 \pm 0.486$ for 72 hours, respectively 
(Figure 1). During Tan-IIA treatment for 24, 48 and 72 hours, the half-maximum inhibitory concentration (IC50) was $12.43,4.57$ and $2.47 \mu \mathrm{M}$, respectively.

\subsection{The Effect of Tan-IIA on the Protein Expressions of Bip, PDI, Calnexin, and Calreticulin in BxPC-3 Cells}

BxPC-3 cells were treated with various concentrations $(0,4.2$ and $8.5 \mu \mathrm{M})$ of Tan-IIA for 24 hours and the proteins expression levels were evaluated by western blotting. The results showed that Tan-IIA decreased the protein expression levels of Bip, PDI, Calnexin and Calreticulin (Figure 2(a)). BxPC-3 cells were treated with various concentrations $(0,2.0$ and $4.0 \mu \mathrm{M})$ of Tan-IIA for 48 hours and the proteins expression levels were evaluated by western blotting. The results showed that Tan-IIA decreased the protein expression levels of Bip, PDI, Calnexin and Calreticulin (Figure 2(b)). BxPC-3 cells were treated with Tan-IIA $(8.5 \mu \mathrm{M})$ for different durations $(0,24,48$ and $72 \mathrm{~h})$ and then the proteins expression levels were evaluated by western blotting. The results showed that Tan-IIA decreased the protein expression levels of Bip, PDI and Calnexin but mild increased Calreticulin expression (Figure 3). BxPC-3 cells were treated with Tan-IIA $(8.5 \mu \mathrm{M})$ for different durations $(0,2,4,6$, 8, 12, 24 and $48 \mathrm{~h}$ ) and then the proteins expression levels of Bip, PDI, Calnexin, Calreticulin were evaluated by western blotting. The results showed that the protein expression levels of Bip (Figure 4(a)), PDI (Figure 4(b)), Calnexin (Figure 4(c)) and Calreticulin (Figure 4(d)) are decreased in BxPC-3 cells were treated with Tan-IIA.

\subsection{The Effect of Tan-IIA on the Protein Expressions of IRE1 $\alpha$, PERK, elF2 $\alpha$, ATF6, ATF4, Caspase-12, Caspase-9, CHOP and Bcl-2 in BxPC-3 Cells}

BxPC-3 cells were treated with various concentrations $(0,4.2$ and $8.5 \mu \mathrm{M})$ of Tan-IIA for 24 hours and the proteins expression levels were evaluated by western blotting. The results showed that Tan-IIA increased the protein expression levels of IRE1 $\alpha$, PERK, elF2 $\alpha$, ATF6, CHOP, Caspase-12, Caspase-9 and Caspase-3 (Figure 5(a)); but decrease Bcl-2 (Figure 5(b)). BxPC-3 cells were treated with various concentrations (0, 2.0 and 4.0 $\mu \mathrm{M})$ of Tan-IIA for 48 hours and the proteins expression levels were evaluated by western blotting. The results showed that Tan-IIA increased the protein expression levels of IRE1 $\alpha$, PERK, elF2 $\alpha$, ATF6, CHOP, Caspase-12,

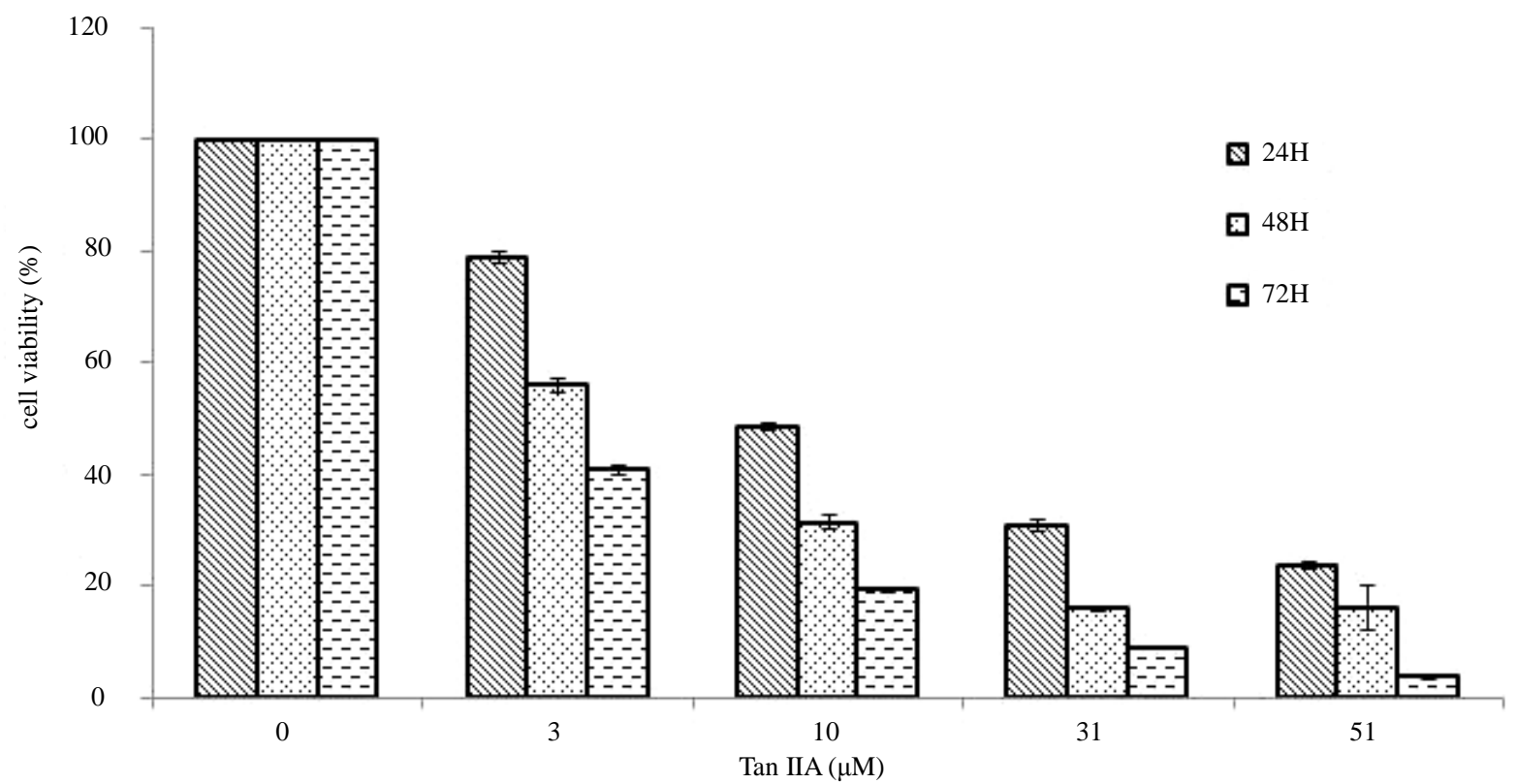

Figure 1. The cytotoxicity of Tanshinone IIA BxPC-3 cells were cultured with various concentrations $(0,3,10,31$ and 51 $\mu \mathrm{M})$ of Tan-IIA for different durations (24, 48 and 72 hours). The results showed that during Tan-IIA treatment for 24, 48 and 72 hours, the half-maximum inhibitory concentration (IC50) was $12.43,4.57$ and $2.47 \mu \mathrm{M}$, respectively. Each point is the mean \pm SD of three experiments. 
Tan IIA $(\mu \mathrm{M})$

$4.2 \quad 8.5$

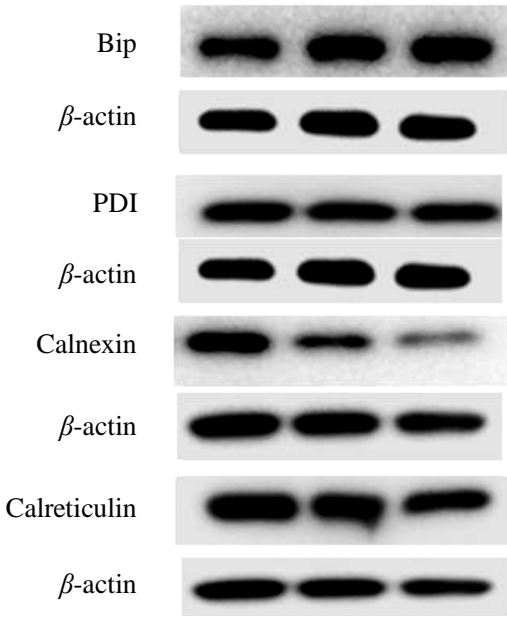

(a)

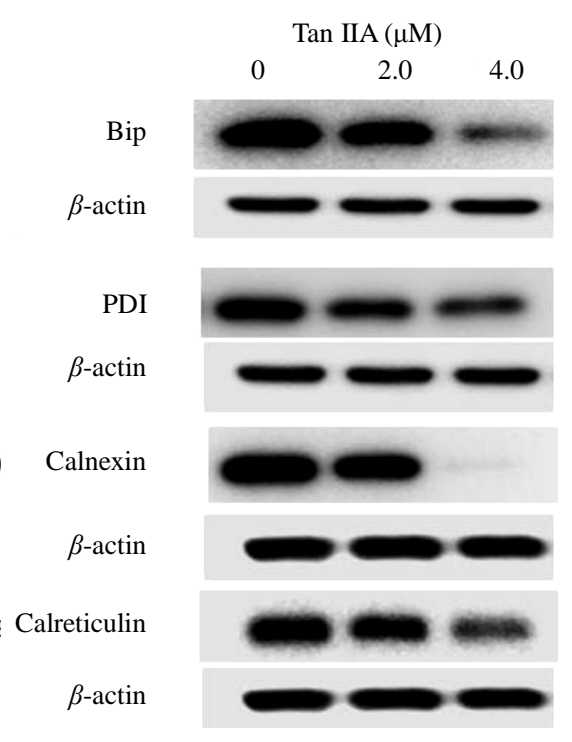

(b)

Figure 2. The protein expression of Bip, PDI, Calnexin, and Calreticulin in BxPC-3 cells BxPC-3 cells were treated with various concentrations $(0,4.2$ and $8.5 \mu \mathrm{M})$ of Tan-IIA for 24 hours or $(0,2.0$ and $4.0 \mu \mathrm{M})$ of Tan-IIA for 48 hours, and then the proteins expression levels were evaluated by western blotting. The results showed that Tan-IIA decreased the protein expression levels of Bip, PDI, Calnexin and Calreticulin (Figure 2(a), Figure 2(b)).

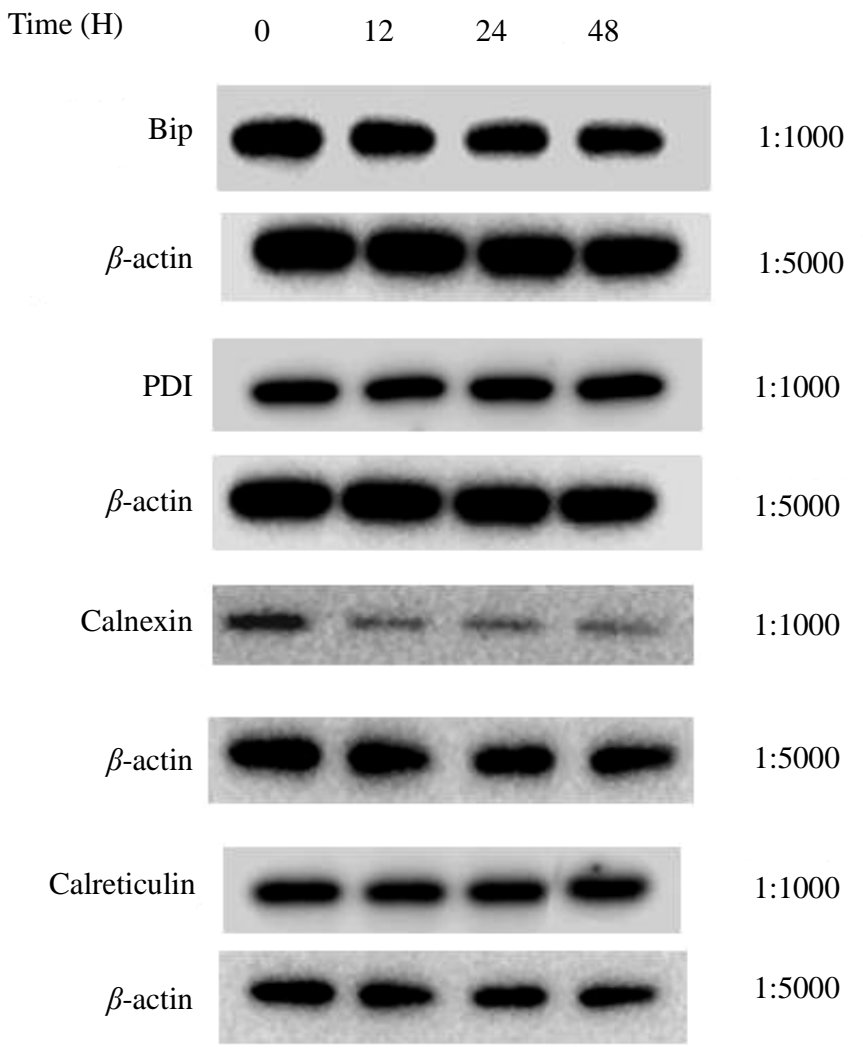

Figure 3. The protein expression of Bip, PDI, Calnexin, and Calreticulin in BxPC-3 cells BxPC-3 cells were treated with Tan-IIA $(8.5 \mu \mathrm{M})$ for $0,24,48$ and $72 \mathrm{~h}$, and then were evaluated by western blotting. The results showed that Tan-IIA decreased the protein expression levels of Bip, PDI and Calnexin but mild increased Calreticulin expression (Figure 3). 


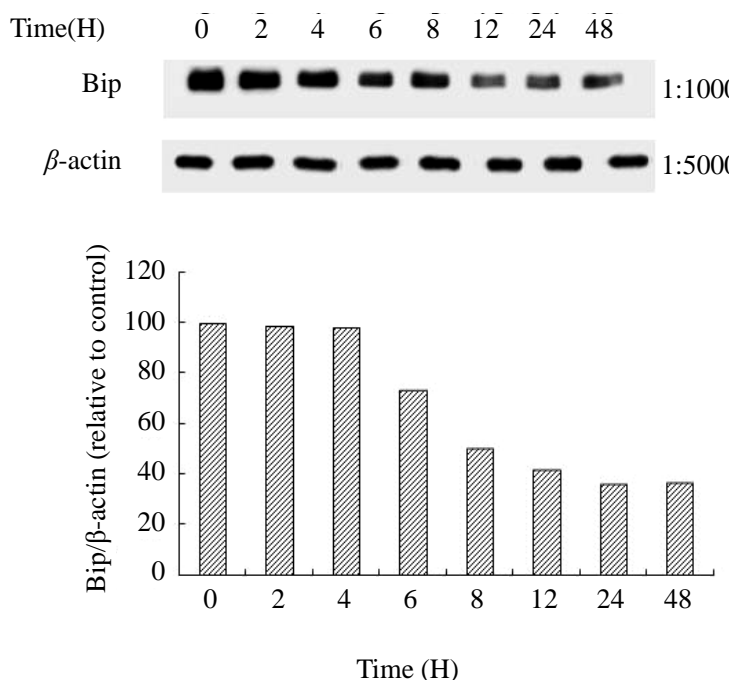

(a)
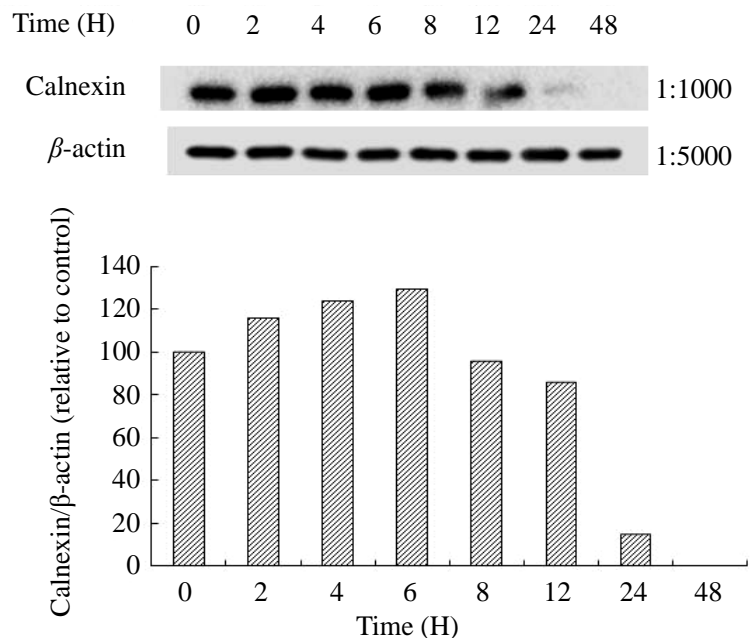

(c)
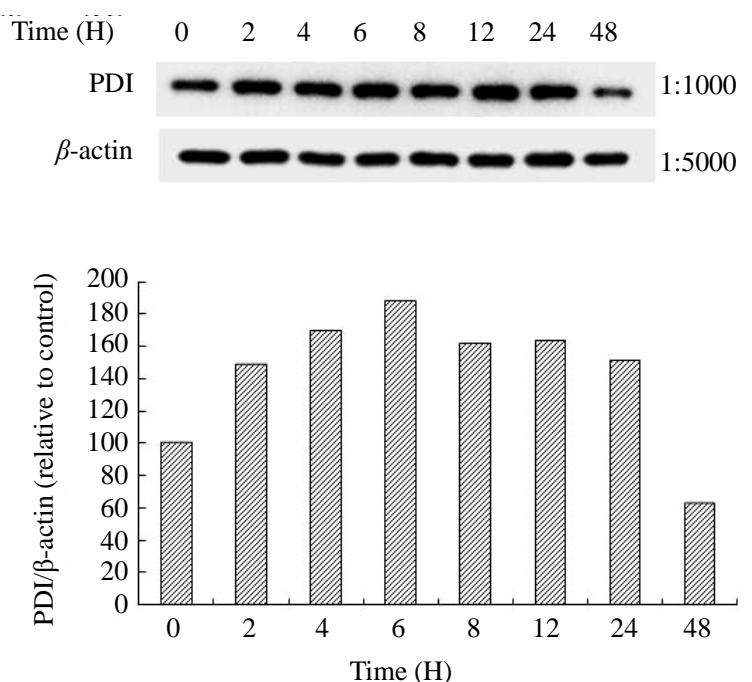

(b)
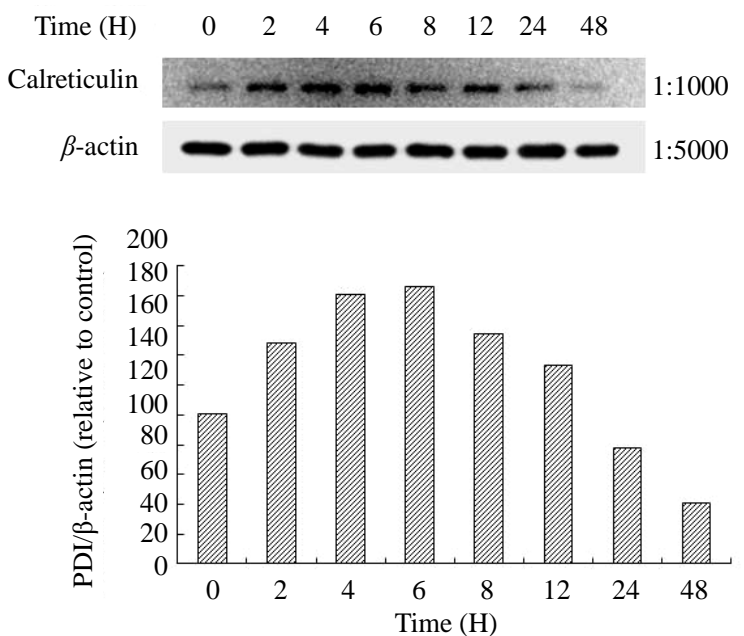

(d)

Figure 4. The protein expression of Bip, PDI, Calnexin, and Calreticulin in BxPC-3 cells BxPC-3 cells were treated with Tan-IIA $(8.5 \mu \mathrm{M})$ for different durations $(0,2,4,6,8,12,24$ and $48 \mathrm{~h})$ and then the proteins expression levels of Bip, PDI, Calnexin, Calreticulin were evaluated by western blotting. The results showed that the protein expression levels of Bip (Figure 4(a)), PDI (Figure 4(b)), Calnexin (Figure 4(c)) and Calreticulin (Figure 4(d)) are decreased in BxPC-3 cells were treated with Tan-IIA.

Caspase-9 and Caspase-3 (Figure 6(a)); but decrease Bcl-2 (Figure 6(b)). BxPC-3 cells were treated with TanIIA $(8.5 \mu \mathrm{M})$ for different durations $(0,24,48$ and $72 \mathrm{~h})$ and then the proteins expression levels were evaluated by western blotting. The results showed that Tan-IIA increased the protein expression levels of PERK, elF2 $\alpha$, ATF4, IRE1 $\alpha$, ATF6, CHOP, Caspase-12 and Caspase-9 (Figure 6(a)), but decreased p-JNK (data no show) and Bcl-2 (Figure 6(b)).

\subsection{The Protein Expression of Caspase-3 in BxPC-3 Cells}

BxPC-3 cells were treated with various concentrations $(0,4.2$ and $8.5 \mu \mathrm{M})$ of Tan-IIA for 24 hours. The protein expression of Caspase-3 in BxPC-3 cells was observed by immunocytochemistry as described in materials and methods. The results showed that Tan-IIA could induce high level of Caspase-3 (Figure 7).

\section{Discussion}

Our results showed that Tan-IIA can inhibit the proliferation of human pancreatic cancer BxPC-3 cells with 


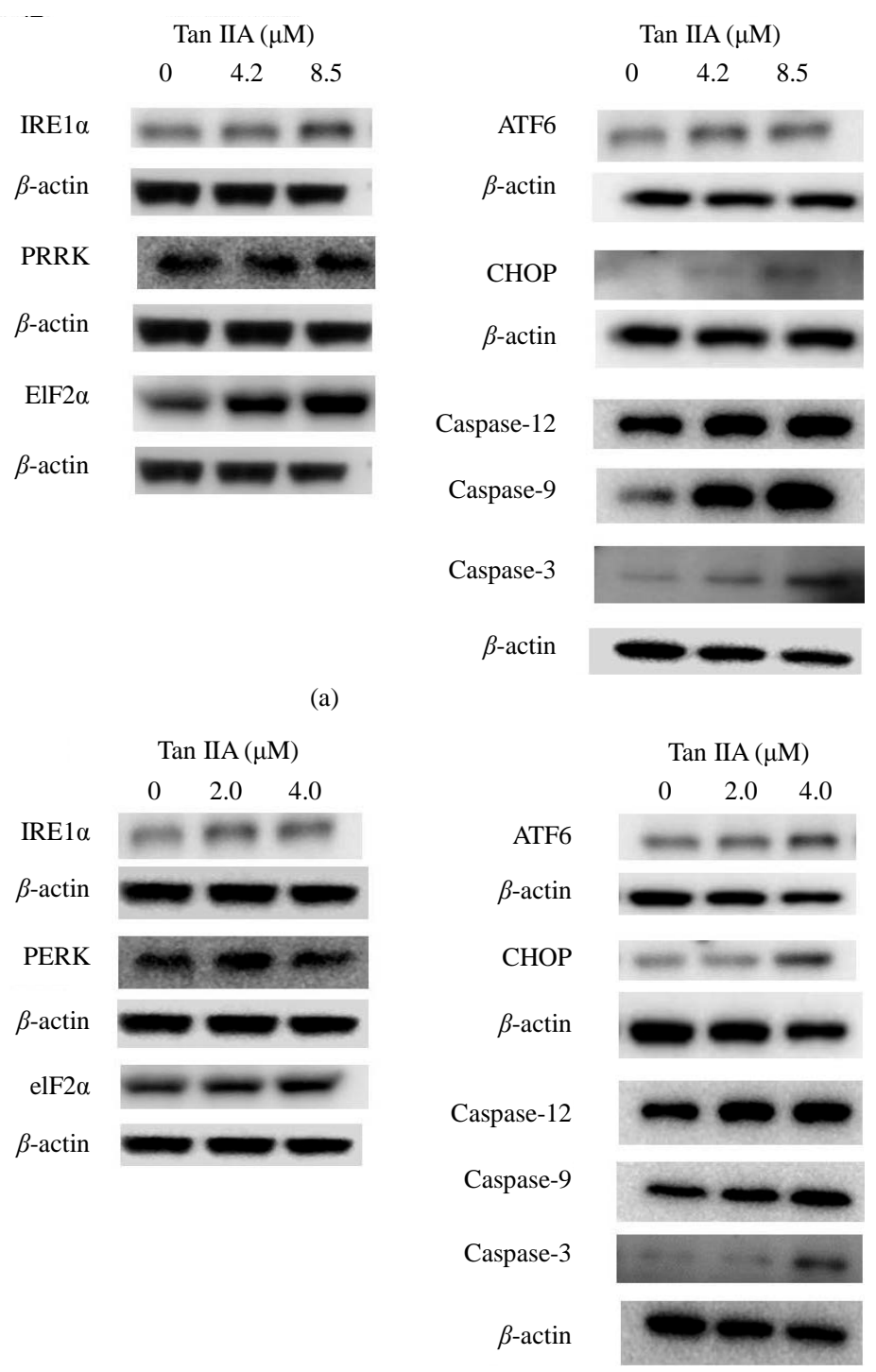

(c)

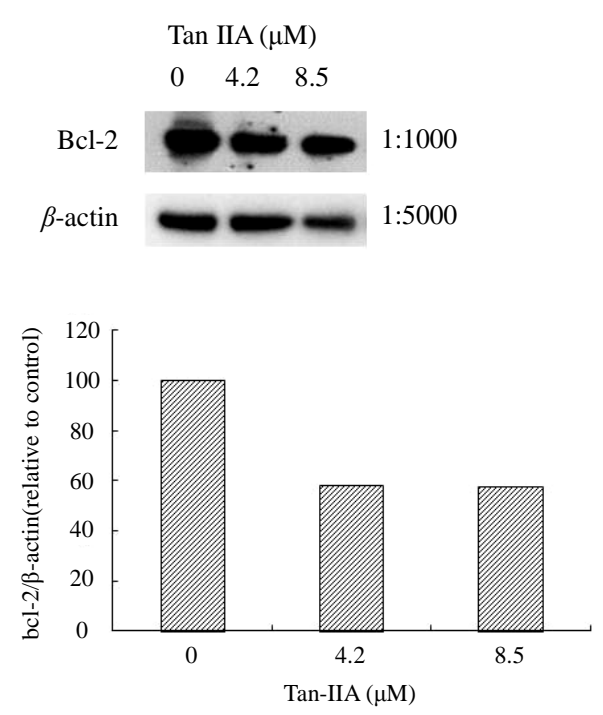

(b)
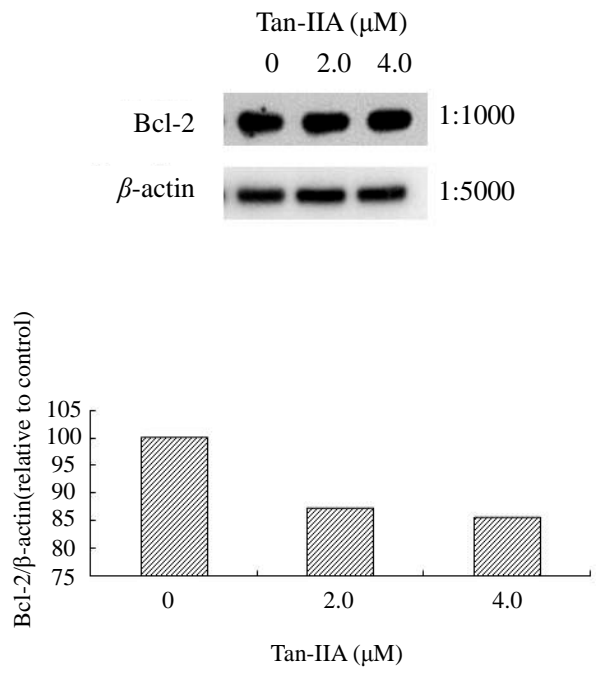

(d)

Figure 5. The effects of Tan-IIA on the protein expression of IRE1 $\alpha$, PERK, and elF2 $\alpha$, ATF6, ATF4, Caspase-12, Caspase-9, CHOP and Bcl-2 in BxPC-3 cells BxPC-3 cells were treated with various concentrations $(0,4.2$ and $8.5 \mu \mathrm{M})$ of Tan-IIA for 24 hours or $(0,2.0$ and $4.0 \mu \mathrm{M})$ of Tan-IIA for 48 hours, and then the proteins expression levels were evaluated by western blotting. The results showed that Tan-IIA increased the protein expression levels of IRE1 $\alpha$, PERK, elF2 $\alpha$, ATF6, CHOP, Caspase-12, Caspase-9 and Caspase-3 (Figure 5(a), Figure 5(c)); but decrease Bcl-2 (Figure 5(b), Figure 5(d)).

time- and dose-dependent in vitro (Figure 1). This is agreement with other documents [16] [19]. It is well documented that decrease the protein expressions related to unfolding protein response (UPR) will inducing ER stress [23] [24]. Our results showed that the protein expression levels of Bip (Figure 4(a)), PDI (Figure 4(b)), Calnexin (Figure 4(c)) and Calreticulin (Figure 4(d)) are decreased in BxPC-3 cells were treated with Tan-IIA. These findings indicate Tan-IIA can induce ER stress in BxPC-3 cells. It is well documented for the activation of ER stress in cancer therapy. When upstream elements of ER stress (such as IRE1 $\alpha$, PERK, Caspase-12 and ATF6) were activated and then the target proteins CHOP was increased [25]. The target proteins CHOP could inhibit Bcl-2 expression was well documented. When UPR exceed the threshold, these cells were damaged and may be through inducing ATF6 mediate CHOP signaling pathway to induce apoptosis [26] [27]. Our result also showed that BxPC-3 cells were treated with Tan-IIA can increase PERK, elF2 $\alpha$, ATF4, IRE1 $\alpha$, ATF6, CHOP, Caspase-12 and Caspase-9 (Figure 6(a)) expression but decrease Bcl-2 (Figure 6(b)) expression with time and dose dependent. The Immunocy to chemistry results also showed that Tan-IIA could induce high level of 


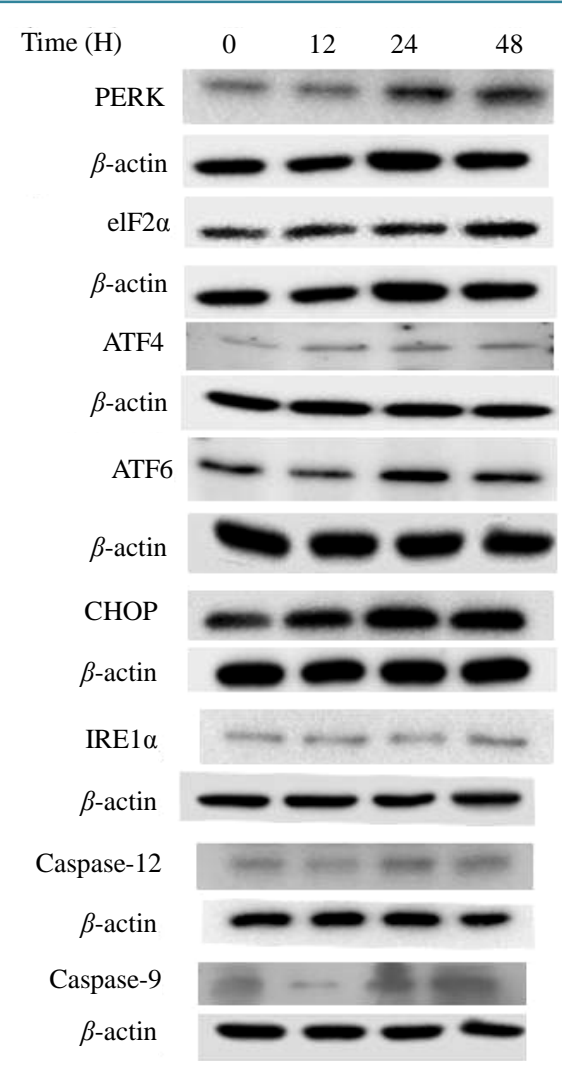

(a)

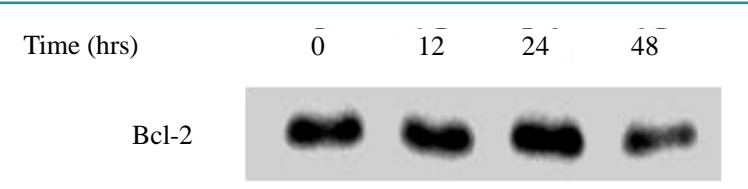

$\beta$-actin

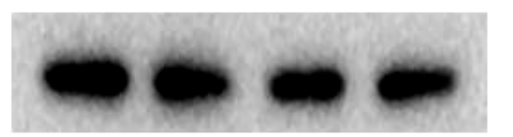

1:5000

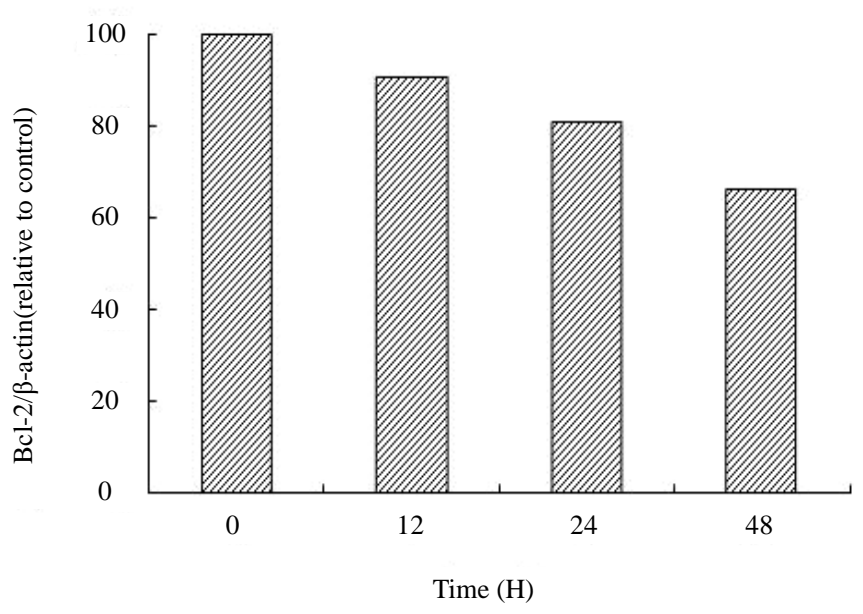

(b)

Figure 6. The effects of Tan-IIA on the protein expression of IRE1 $\alpha$, PERK, elF2 $\alpha$, ATF6, ATF4, Caspase-12, Caspase-9, CHOP and Bcl-2 in BxPC-3 cells BxPC-3 cells were treated with Tan-IIA $(8.5 \mu \mathrm{M})$ for different durations $(0,24,48$ and 72 h) and then the proteins expression levels were evaluated by western blotting. The results showed that Tan-IIA increased the protein expression levels of PERK, elF2 $\alpha$, ATF4, IRE1 $\alpha$, ATF6, CHOP, Caspase-12 and Caspase-9 (Figure 6(a)), but decreased p-JNK (data no show) and Bcl-2 (Figure 6(b)).

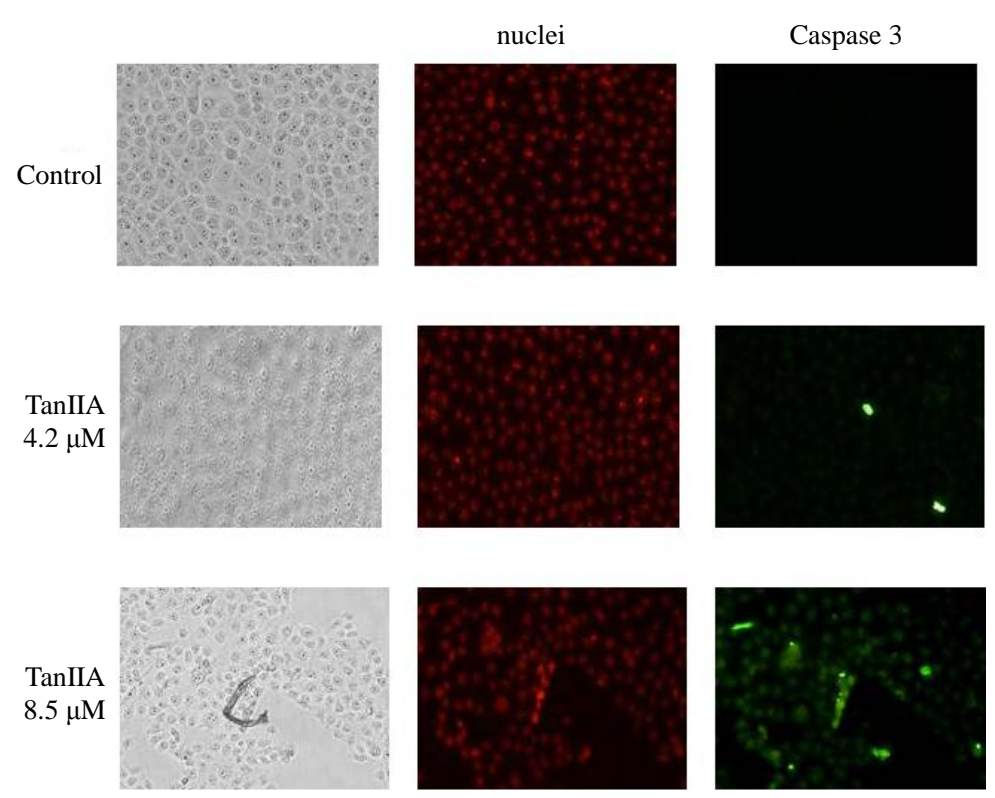

Figure 7. The protein expression of Caspase-3 in BxPC-3 cells BxPC-3 cells were treated with Tan-IIA (0, 4.2 and 8.5 $\mu \mathrm{M})$ for 24 hours. The protein expression of Caspase-3 in BxPC-3 cells was observed by immunocytochemistry as described in materials and methods. The results showed that Tan-IIA could induce high level of Caspase-3 (Figure 7). 


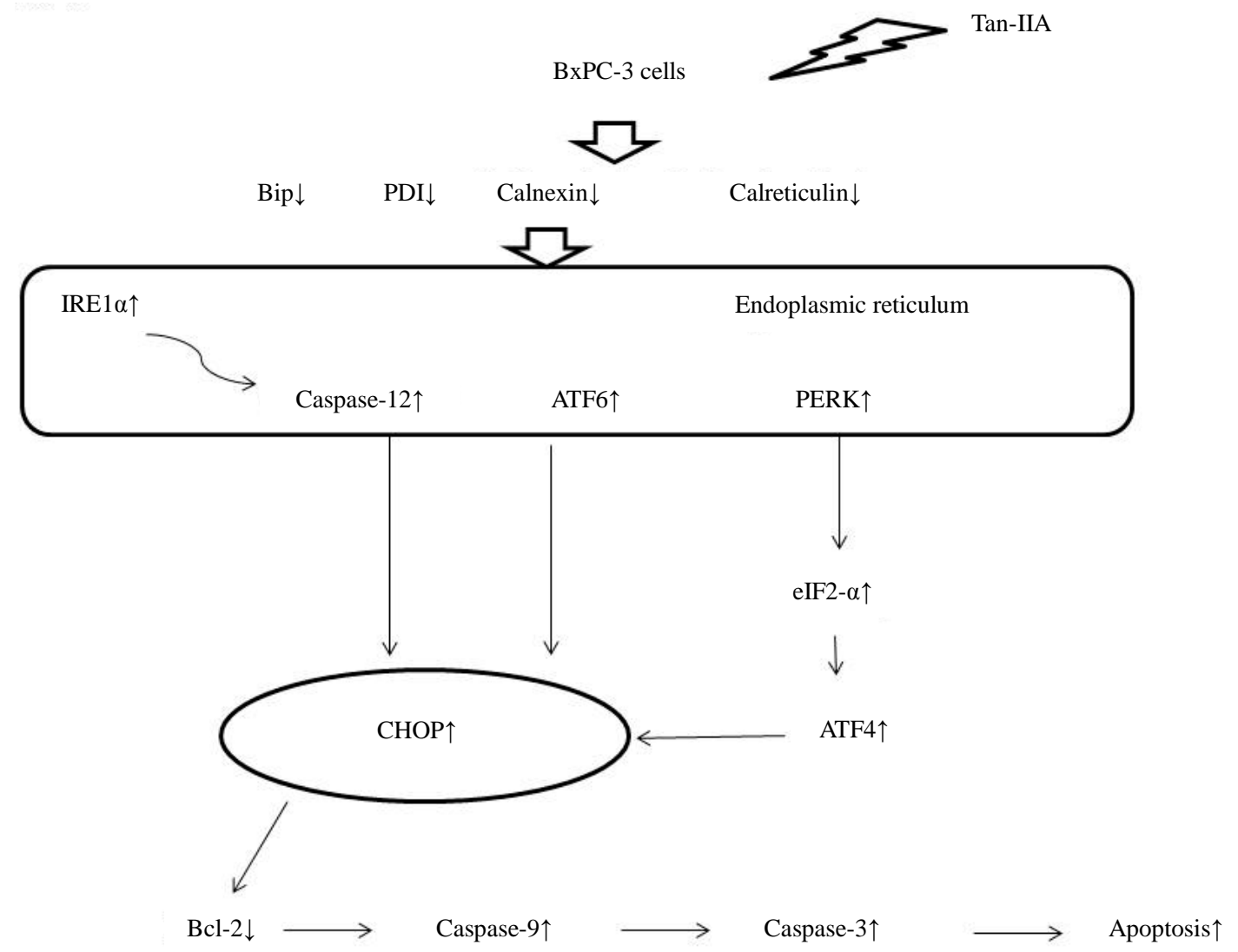

Figure 8. The proposed molecular mechanisms for Tan-IIA to inhibited BxPC-3 cells Tan-IIA induce UPR through decreasing the protein expression of Bip, PDI, Calnexin and Calreticulin, then increase PERK, elF $2 \alpha$, ATF4, IRE1 $\alpha$, Caspase-12 and ATF6 expression and then stimuli ER stress downstream CHOP over expression. In addition, CHOP decreased Bcl-2 protein expression and induced mitochondria dysfunction to induce apoptosis (Figure 8).

Caspase-3 (Figure 7). These findings indicate that Tan-IIA could induce apoptosis through inducing ER stress in BxPC-3 cells in vitro. This is the first report for Tan-IIA could inhibit the proliferations of human pancreatic cancer BxPC-3 cells through ER stress pathway. Tan-IIA with chemotherapeutic potential for human pancreatic cancer BxPC-3 cells in vitro. It is warrants further study in the future. The proposed molecular mechanisms for Tan-IIA to inhibited BxPC-3 cells as follow (Figure 8): Tan-IIA induce UPR through decreasing the protein expression of Bip, PDI, Calnexin and Calreticulin, then increase PERK, elF2 $\alpha$, ATF4, IRE1 $\alpha$, Caspase-12 and ATF6 expression, and then stimuli ER stress downstream CHOP over expression. In addition, CHOP decreased Bcl-2 protein expression and induced mitochondria dysfunction to induce apoptosis.

\section{Conflict of Interest Statement}

No competing interests.

\section{Authors' Contributions}

Chin-Cheng Su is the single investigator for this study providing oversight and contributed fundamental conceptualization for the research, writing a grant proposal and manuscript.

\section{Acknowledgements}

This study was supported by grant 101-CCH-IRP-11 from the Research Section of the Changhua Christian Hospital, Changhua, Taiwan Province, China. 


\section{References}

[1] Siegel, R., Naishadham, D. and Jemal, A. (2012) Cancer Statistics. CA-A Cancer Journal for Clinicians, 62, 10-29. http://dx.doi.org/10.3322/caac.20138

[2] Burris 3rd, H.A., Moore, M.J., Andersen, J., et al. (1997) Improvements in Survival and Clinical Benefit with Gemcitabine as First-Line Therapy for Patients with Advanced Pancreas Cancer: A Randomized Trial. Journal of Clinical Oncology, 15, 2403-2413.

[3] Heinemann, V., Quietzsch, D., Gieseler, F., et al. (2006) Randomized Phase III Trial of Gemcitabine plus Cisplatin Compared with Gemcitabine Alone in Advanced Pancreatic Cancer. Journal of Clinical Oncology, 24, 3946-3952. http://dx.doi.org/10.1200/JCO.2005.05.1490

[4] Louvet, C., Labianca, R., Hammel, P., et al. (2005) Gemcitabine in Combination with Oxaliplatin Compared with Gemcitabine Alone in Locally Advanced or Metastatic Pancreatic Cancer: Results of a GERCOR and GISCAD Phase III Trial. Journal of Clinical Oncology, 23, 3509-3516. http://dx.doi.org/10.1200/JCO.2005.06.023

[5] Herrmann, R., Bodoky, G., Ruhstaller, T., et al. (2007) Gemcitabine plus Capecitabine Compared with Gemcitabine Alone in Advanced Pancreatic Cancer: A Randomized, Multicenter, Phase III Trial of the Swiss Group for Clinical Cancer Research and the Central European Cooperative Oncology Group. Journal of Clinical Oncology, 25, 22122217. http://dx.doi.org/10.1200/JCO.2006.09.0886

[6] Che, A.J., Zhang, J.Y., Li, C.H., Chen, X.F., Hu, Z.D. and Chen, X.G. (2004) Separation and Determination of Active Components in Radix Salviae Miltiorrhizae and Its Medicinal Preparations by Nonaqueous Capillary Electrophoresis. Journal of Separation Science, 27, 569-575. http://dx.doi.org/10.1002/jssc.200301710

[7] Zhou, L., Zuo, Z. and Chow, M.S. (2005) Danshen: An Overview of Its Chemistry, Pharmacology, Pharmacokinetics, and Clinical Use. Journal of Clinical Pharmacology, 45, 1345-1359. http://dx.doi.org/10.1177/0091270005282630

[8] Jang, S.I., Kim, H.J., Kim, Y.J., Jeong, S.I. and You, Y.O. (2006) Tanshinone IIA Inhibits LPS Induced NF-KappaB Activation in RAW 264.7 Cells: Possible Involvement of the NIK-IKK, ERK1/2, p38 and JNK Pathways. European Journal of Pharmacology, 542, 1-7. http://dx.doi.org/10.1016/j.ejphar.2006.04.044

[9] Li, W., Li, J., Ashok, M., Wu, R., Chen, D., Yang, L., et al. (2007) A Cardiovascular Drug Rescues Mice from Lethal Sepsis by selectively Attenuating a Late-Acting Pro-Inflammatory Mediator, High Mobility Group Box 1. Journal of Immunology, 78, 3856-3864. http://dx.doi.org/10.4049/jimmunol.178.6.3856

[10] Lin, R., Wang, W.R., Liu, J.T., Yang, G.D. and Han, C.J. (2006) Protective Effect of Tanshinone IIA on Human Umbilical Vein Endothelial Cell Injured by Hydrogen Peroxide and Its Mechanism. Journal of Ethnopharmacology, 108, 217-222. http://dx.doi.org/10.1016/j.jep.2006.05.004

[11] Wang, A.M., Sha, S.H., Lesniak, W. and Schacht, J. (2003) Tanshinone (Salviae miltiorrhizae Extract) Preparations Attenuate Aminoglycoside-Induced Free Radical Formation in Vitro and Ototoxicity in Vivo. Antimicrobial Agents and Chemotherapy, 47, 1836-1841. http://dx.doi.org/10.1128/AAC.47.6.1836-1841.2003

[12] Su, C.C. and Lin, Y.H. (2008) Tanshinone IIA Down-Regulates the Protein Expression of ErbB-2 and Up-Regulates TNF-Alpha in Colon Cancer Cells in Vitro and in Vivo. International Journal of Molecular Medicine, 22, 847-851.

[13] Su, C.C. and Lin, Y.H. (2008) Tanshinone IIA Inhibits Human Breast Cancer Cells through Increased Bax to Bcl-xL Ratios. International Journal of Molecular Medicine, 22, 357-361.

[14] Chiu, T.L. and Su, C.C. (2010) Tanshinone IIA Induces Apoptosis in Human Lung Cancer A549 Cells through the Induction of Reactive Oxygen Species and Decreasing the Mitochondrial Membrane Potential. International Journal of Molecular Medicine, 25, 231-236.

[15] Cheng, C.Y. and Su, C.C. (2010) Tanshinone IIA May Inhibit the Growth of Small Cell Lung Cancer H146 Cells by Up-Regulating the Bax/Bcl-2 Ratio and Decreasing Mitochondrial Membrane Potential. Molecular Medicine Reports, 3, 645-650.

[16] Fronza, M., Murillo, R., Ślusarczyk, S., Adams, M., Hamburger, M., Heinzmann, B., Laufer, S. and Merfort, I. (2011) In Vitro Cytotoxic Activity of Abietane Diterpenes from Peltodon longipes as well as Salvia miltiorrhiza and Salvia sahendica. Bioorganic \& Medicinal Chemistry, 19, 4876-4881. http://dx.doi.org/10.1016/j.bmc.2011.06.067

[17] Cheng, C.Y. and Su, C.C. (2010) Tanshinone IIA Inhibits Hep-J5 Cells by Increasing Calreticulin, Caspase 12 and GADD153 Protein Expression. International Journal of Molecular Medicine, 26, 379-385.

[18] Yan, M.Y., Chien, S.Y., Ku, S.J., Chen, D.R. and Su, C.C. (2012) Tanshinone IIA Inhibits BT-20 Human Breast Cancer Cell Proliferation through Increasing Caspase 12, GADD153 and Phospho-p38 Protein Expression. International Journal of Molecular Medicine, 29, 855-863.

[19] Huang, C.Y., Chiu, T.L., Kuo, S.J., Chien, S.Y., Chen, D.R. and Su, C.C. (2013) Tanshinone IIA Inhibits the Growth of Pancreatic Cancer BxPC-3 Cells by Decreasing Protein Expression of TCTP, MCL-1 and Bcl-xL. Molecular Medicine Report, 7, 1045-1049. 
[20] Mossman, T. (1983) Rapid Colorimetric Assay for Cellular Growth and Survival: Application to Proliferation and Cytotoxicity Assays. Journal of Immunological Methods, 65, 55-63. http://dx.doi.org/10.1016/0022-1759(83)90303-4

[21] Chen, H.C., Hsieh, W.T., Chang, W.C. and Chung, J.G. (2004) Aloe-Emodin Induced in Vitro G2/M Arrest of Cell Cycle in Human Promyelocytic Leukemia HL-60 Cells. Food and Chemical Toxicology, 42, 1251-1257. http://dx.doi.org/10.1016/j.fct.2004.03.002

[22] Bradford, M.M. (1976) A Rapid and Sensitive Method for the Quantization of Microgram Quantities of Protein Using the Principle of Protein-Dye Binding. Analytical Biochemistry, 72, 248-254. http://dx.doi.org/10.1016/0003-2697(76)90527-3

[23] Kaufman, R.J., Scheuner, D., Schroder, M., Shen, X., Lee, K., Liu, C.Y. and Arnold, S.M. (2002) The Unfolded Protein Response in Nutrient Sensing and Differentiation. Nature Reviews Molecular Cell Biology, 3, 411-421. http://dx.doi.org/10.1038/nrm829

[24] Wu, J. and Kaufman, R.J. (2006) From Acute ER Stress to Physiological Roles of the Unfolded Protein Response. Cell Death Differentiation, 13, 374-384. http://dx.doi.org/10.1038/sj.cdd.4401840

[25] Ma, Y. and Hendershot, L.M. (2004) The Role of the Unfolded Protein Response in Tumour Development: Friend or Foe? Nature Reviews Cancer, 4, 966-977. http://dx.doi.org/10.1038/nrc1505

[26] Kim, R., Emi, M., Tanabe, K. and Murakami, S. (2006) Role of the Unfolded Protein Response in Cell Death. Apoptosis, 11, 5-13. http://dx.doi.org/10.1007/s10495-005-3088-0

[27] Rasheva, V.I. and Domingos, P.M. (2009) Cellular Responses to Endoplasmic Reticulum Stress and Apoptosis. Apoptosis, 14, 996-1007. http://dx.doi.org/10.1007/s10495-009-0341-y

\section{Abbreviations}

Tan-IIA: Tanshinone IIA;

ATF6: Activating transcription factor-6;

ein-1(IRE1 $\alpha$ ): Iinositol-requiring protein kinase RNA (PKR)-like ER kinase;

PERK: Protein kinase RNA (PKR)-like ER kinase;

CHOP: Also known as GADD153, C/EBP-homologous protein;

FBS: Fetal bovine serum;

ER stress: Endoplasmic reticulum stress. 